\section{Retroalimentación integral (debriefing) oral y asistida por video en simulación de reanimación cardiopulmonar avanzada: estudio piloto}

Edgar Luna-Villanueva, Moisés de los Santos-Rodríguez, Gilberto Sierra Basto, Carlos R. González-Arriaga, Fanny G. Zamora-Graniel

Introducción. En los cursos de soporte vital básico y avanzado con simuladores, parte del proceso de aprendizaje incluye la retroalimentación integral (debriefing), que consiste en un momento de reflexión individual o en equipo acerca de lo que sucedió durante el escenario de simulación y lo que significó para cada miembro.

Objetivo. Comparar debriefing oral con o sin video en el logro de la competencia de reanimación cardiopulmonar (RCP) en modelos de simulación.

Sujetos y métodos. Estudio piloto experimental (antes y después) con 30 estudiantes del quinto curso de la licenciatura de médico cirujano de la Facultad de Medicina de la Universidad Autónoma de Yucatán, aleatorizados en dos grupos: 15 alumnos que recibieron debriefing oral (grupo I) y 15 que recibieron debriefing asistido por video (grupo II) de la sesión de simulación. Cada sesión de retroalimentación tuvo una duración aproximada de 20 minutos.

Resultados. Los resultados mostraron mejoría de los pasos de RCP: intervenciones y abordaje grupo I, 3 (20\%); liderazgo, 4 (27\%); manejo del ritmo inicial del paro, 3 (20\%); calidad de la RCP avanzada, 11 (73\%); cuidados posparada, $5(33 \%)$; logro de la competencia, $5(33 \%)$; grupo II: 7 (47\%), 14 (93\%), $12(80 \%), 13(87 \%), 14(93 \%)$ y $13(87 \%)$, respectivamente, con una diferencia significativa (prueba exacta de Fisher) en liderazgo, manejo del ritmo inicial, cuidados posparada y logro de la competencia.

Conclusiones. Hay ventaja del debriefing asistido por video en las habilidades no técnicas y en el dominio de la competencia de RCP avanzada.

Palabras clave. Modelos de simulación. Reanimación cardiopulmonar. Retroalimentación.

\section{Oral and video-assisted debriefing in advanced cardiopulmonary resuscitation simulation: a pilot study}

Introduction. Part of the learning process of the simulator courses of basic and advanced life support includes debriefing, which is a time for individual, or team reflection, about what happened during the simulation scenario, and what it meant for each member.

Aim. To compare the oral debriefing, with or without video, in achieving competence in cardiopulmonary resuscitation (CPR) on simulation models.

Subjects and methods. Experimental study. A group of 30 students in the fifth year of the School of Medicine of Universidad Autónoma de Yucatán, were selected. Participants were randomly divided into two groups: 15 students who received oral debriefing (OD) and 15 students who received video-assisted debriefing training (VD), each session lasting 20 minutes.

Results. Results showed improvement in the following steps: interventions and approach OD 3 (20\%) and VD 7 (47\%), leadership OD 4 (27\%) and VD 14 (93\%), initial rhythm handling OD $3(20 \%)$ and VD 12 (80\%), advanced CPR quality OD 11 (73\%) and VD 13 (87\%), post-cardiac arrest care OD 5 (33\%) and VD 14 (93\%), and achieving competition OD 5 (33\%) and VD $13(87 \%)$. Significant differences using Fisher's exact test were found in leadership, initial rhythm handling, postcardiac arrest care and achieving competition.

Conclusions. Students achieve better skills on advanced CPR after simulation model training with video-assisted debriefing. Key words. Cardiopulmonary resuscitation. Debriefing. Simulation models.
Departamento para el Entrenamiento de las Competencias Disciplinarias del Área de la Salud (DECODAS). Facultad de Medicina. Universidad Autónoma de Yucatán. Mérida, Yucatán, México.

Correspondencia: MC. Edgar Luna Villanueva. DECODAS. Facultad de Medicina. Universidad Autónoma de Yucatán. Avda. Itzaes, 498, 59 y 59A. Col. Centro. CP 97000. Mérida, Yucatán, México.

E-mail: edgluna@hotmail.com

Agradecimientos:

A los Dres. J.J. Pérez, P.M. González, C.R. Ojeda y E. Lubcke, así como a los estudiantes de la Facultad de Medicina de la UADY, por su apoyo y participación incondicional durante el desarrollo de este proyecto.

Conflicto de intereses: No declarado.

Competing interests: None declared.

(c) 2015 FEM 


\section{Introducción}

En la enseñanza de la reanimación cardiopulmonar (RCP) básica y avanzada es común el empleo de simuladores. Según el grado de semejanza con la realidad que ofrezcan, presentarán un determinado nivel de fidelidad, algunos de ellos con un nivel elevado al reproducir características de un ser humano real [1-3]. Se han demostrado las ventajas de los cursos teórico-prácticos contra los cursos sólo teóricos [4]. En algunos estudios se ha observado que un mayor nivel de fidelidad del maniquí empleado en la simulación guarda relación con un mayor logro de las competencias en RCP y un mayor desarrollo de las aptitudes de liderazgo y mayor confianza [5,6], aunque otros estudios no muestran diferencias significativas [7].

Además de la fidelidad empleada en el entrenamiento basado en la simulación, es posible valerse de otras estrategias de manera alternativa para aumentar la retención y la adquisición de habilidades en RCP. Algunas de estas estrategias son las listas de cotejo y algoritmos, dispositivos de retroalimentación como indicadores de voz, metrónomos o medidores de la calidad de las compresiones incluidos en los maniquíes, y la retroalimentación integral (debriefing) [8-11].

La retroalimentación integral es una estrategia de enseñanza -que se ha descrito como una parte esencial de la educación médica basada en la simulación- consistente en un momento de reflexión individual o en equipo acerca de lo sucedido durante el escenario de simulación y lo que significó para cada miembro. Esta estrategia se centra en los alumnos y sus experiencias (de manera individual o colectiva) y se emplea para mejorar el desempeño en experiencias o situaciones futuras [1,12-14].

Además de la retroalimentación integral oral, otras estrategias pueden sumarse para aumentar el aprendizaje y logro de competencias en RCP, como presentaciones con diapositivas mediante ordenador o la retroalimentación integral asistida por video $(v i$ deo-assisted debriefing) $[1,12,13,15]$. Aunque se ha estudiado ampliamente, los resultados obtenidos sobre esta técnica no son determinantes [16-19].

\section{Sujetos y métodos}

Estudio piloto experimental, analítico, prospectivo, longitudinal (antes y después), cuantitativo y cualitativo, acerca del desempeño logrado por un grupo de 30 alumnos del quinto año de la licenciatura de médico cirujano [8] durante su Taller de Reanima- ción Pulmonar y Manejo de la Vía Aérea de la Facultad de Medicina.

Se asignaron los 30 alumnos participantes de manera aleatorizada en dos grupos: un grupo control, formado por 15 alumnos que recibieron debriefing oral, y un grupo experimental, compuesto por otros 15 alumnos, que recibieron debriefing asistido por video de la sesión de simulación (Tabla I).

Todos los alumnos de quinto año recibieron tres sesiones teóricas de dos horas de duración, dos sesiones de demostración de dos horas, una sesión práctica obligatoria de manejo de la vía aérea de una hora y una sesión frente al modelo anatómico de simulación de una hora.

Cada equipo de tres alumnos participó en un total de nueve escenarios de simulación, distribuyendo los roles del equipo de reanimación de acuerdo a lo descrito en la tabla II.

Para las prácticas de simulación se empleó el modelo de alta fidelidad de RCP avanzada Megacode Kelly ${ }^{\circledR}$ de Laerdal, en un escenario clínico de alta fidelidad. Para los trazos electrocardiográficos se utilizó un monitor conectado a un generador de rit$\operatorname{mos}$ ACLS ${ }^{\circledR}$.

Las prácticas se grabaron a través de una cámara web de 1 megapíxel, colocada frente al modelo de simulación, desde un ángulo que permitió grabar con claridad el desempeño del equipo, las órdenes del líder y observar las compresiones y el manejo de la vía aérea.

Al final de cada escenario (sesión de simulación), cada equipo tuvo una sesión de retroalimentación integral. En el grupo control, se brindó únicamente retroalimentación integral oral. El grupo experimental recibió de manera simultánea, durante la retroalimentación integral oral, retroalimentación integral asistida por video, proyectando de manera específica aquellos momentos del video útiles para la reflexión a través de un cañón proyector multimedia. Cada sesión de retroalimentación tuvo una duración aproximada de 20 minutos. Con el fin de evitar variabilidad, un solo instructor (facilitador) se encargó de dar las sesiones teóricas, prácticas, los escenarios de simulación y la retroalimentación tanto oral como asistida por video a ambos grupos de alumnos.

$\mathrm{Al}$ concluir las tres prácticas, un experto en RCP, certificado en soporte cardiovascular vital avanzado por la American Heart Association (AHA), distinto al facilitador y sin conocimiento de las intervenciones realizadas en los grupos, se encargó de evaluar el desempeño de cada alumno en los escenarios de simulación en su papel como líder a través de los videos grabados durante su primera prác- 
Tabla I. Características y diferencias entre los grupos de alumnos.

\begin{tabular}{lcc}
\hline & $\begin{array}{c}\text { Debriefing asistido } \\
\text { por video }(n=15)\end{array}$ & $\begin{array}{c}\text { Debriefing oral } \\
(n=15)\end{array}$ \\
\hline Edad media (años) & $23,20 \pm 0,20$ & $23,07 \pm 0,15$ \\
\hline Hombres & 9 & 7 \\
\hline Mujeres & 6 & 8 \\
\hline Grado académico & Quinto & Quinto \\
\hline $\begin{array}{l}\text { Cursos de reanimación } \\
\text { cardiopulmonar previos }\end{array}$ & No & No \\
\hline $\begin{array}{l}\text { Demostraron logro } \\
\text { de la competencia en } \\
\text { la primera evaluación }\end{array}$ & 0 & 0 \\
\hline
\end{tabular}

tica. Esta evaluación permitió conocer el nivel de competencia de RCP básica y avanzada previo que presentó cada alumno inicialmente.

De la misma manera, se realizó una segunda evaluación empleando los videos grabados durante la tercera práctica de cada alumno en su rol de líder.

La competencia en RCP avanzada se evaluó empleando las listas de comprobación de la AHA. Se midió la mejoría alcanzada por cada alumno entre la primera y la segunda evaluación para cada una de las cinco habilidades que se contemplan dentro de esta lista como necesarias para el logro de la competencia en RCP avanzada: intervenciones de soporte vital básico, liderazgo, manejo del ritmo inicial de parada, calidad de la RCP y cuidados posparada.

Aunque se siguió una metodología AHA para la enseñanza y evaluación, no se aplicaron encuestas de satisfacción ni de evaluación de los instructores a los alumnos, ya que no se consideró un objetivo en el protocolo.

El análisis se efectuó empleando la prueba exacta de Fisher. La tabulación se realizó empleando el programa Microsoft Excel, y el análisis estadístico, el programa Graphpad Prism v. 6.0.

\section{Resultados}

Los 30 sujetos de estudio (14 mujeres y 16 hombres) se distribuyeron de la siguiente manera: ocho mujeres y siete hombres en el grupo control y seis mujeres y nueve hombres en el grupo experimental. No se observaron diferencias significativas entre los resultados obtenidos en mujeres y hombres duran-
Tabla II. Distribución de los escenarios de simulación en cada grupo de reanimación cardiopulmonar.

\begin{tabular}{|c|c|c|c|c|}
\hline & Escenario & Sujeto A & Sujeto B & Sujeto C \\
\hline \multirow{3}{*}{ Práctica 1} & 1 & $\begin{array}{l}\text { Líder: descarga } \\
\text { y medicamentos }\end{array}$ & $\begin{array}{l}\text { Compañero: manejo de la } \\
\text { vía aérea y compresiones }\end{array}$ & $\begin{array}{l}\text { Compañero: manejo de la } \\
\text { vía aérea y compresiones }\end{array}$ \\
\hline & 2 & $\begin{array}{l}\text { Compañero: manejo de la } \\
\text { vía aérea y compresiones }\end{array}$ & $\begin{array}{l}\text { Líder: descarga } \\
\text { y medicamentos }\end{array}$ & $\begin{array}{l}\text { Compañero: manejo de la } \\
\text { vía aérea y compresiones }\end{array}$ \\
\hline & 3 & $\begin{array}{l}\text { Compañero: manejo de la } \\
\text { vía aérea y compresiones }\end{array}$ & $\begin{array}{l}\text { Compañero: manejo de la } \\
\text { vía aérea y compresiones }\end{array}$ & $\begin{array}{l}\text { Líder: descarga } \\
\text { y medicamentos }\end{array}$ \\
\hline \multirow{3}{*}{ Práctica 2} & 4 & $\begin{array}{l}\text { Líder: descarga } \\
\text { y medicamentos }\end{array}$ & $\begin{array}{l}\text { Compañero: manejo de la } \\
\text { vía aérea y compresiones }\end{array}$ & $\begin{array}{l}\text { Compañero: manejo de la } \\
\text { vía aérea y compresiones }\end{array}$ \\
\hline & 5 & $\begin{array}{l}\text { Compañero: manejo de la } \\
\text { vía aérea y compresiones }\end{array}$ & $\begin{array}{l}\text { Líder: descarga } \\
\text { y medicamentos }\end{array}$ & $\begin{array}{l}\text { Compañero: manejo de la } \\
\text { vía aérea y compresiones }\end{array}$ \\
\hline & 6 & $\begin{array}{l}\text { Compañero: manejo de la } \\
\text { vía aérea y compresiones }\end{array}$ & $\begin{array}{l}\text { Compañero: manejo de la } \\
\text { vía aérea y compresiones }\end{array}$ & $\begin{array}{l}\text { Líder: descarga } \\
\text { y medicamentos }\end{array}$ \\
\hline \multirow{3}{*}{ Práctica 3} & 7 & $\begin{array}{l}\text { Líder: descarga } \\
\text { y medicamentos }\end{array}$ & $\begin{array}{l}\text { Compañero: manejo de la } \\
\text { vía aérea y compresiones }\end{array}$ & $\begin{array}{l}\text { Compañero: manejo de la } \\
\text { vía aérea y compresiones }\end{array}$ \\
\hline & 8 & $\begin{array}{l}\text { Compañero: manejo de la } \\
\text { vía aérea y compresiones }\end{array}$ & $\begin{array}{l}\text { Líder: descarga } \\
\text { y medicamentos }\end{array}$ & $\begin{array}{l}\text { Compañero: manejo de la } \\
\text { vía aérea y compresiones }\end{array}$ \\
\hline & 9 & $\begin{array}{l}\text { Compañero: manejo de la } \\
\text { vía aérea y compresiones }\end{array}$ & $\begin{array}{l}\text { Compañero: manejo de la } \\
\text { vía aérea y compresiones }\end{array}$ & $\begin{array}{l}\text { Líder: descarga } \\
\text { y medicamentos }\end{array}$ \\
\hline
\end{tabular}

Tabla III. Evaluación y comparación de los pasos del procedimiento en ambos grupos.

\begin{tabular}{lccc}
\hline & $\begin{array}{c}\text { Mejoría observada } \\
\text { en el grupo de } \\
\text { debriefing oral }\end{array}$ & $\begin{array}{c}\text { Mejoría observada en } \\
\text { el grupo de debriefing } \\
\text { asistido por video }\end{array}$ & $p^{\text {a }}$ \\
\hline Intervenciones y abordaje de RCP básica & $3(20 \%)$ & $7(47 \%)$ & NS \\
\hline Liderazgo & $4(27 \%)$ & $14(93 \%)$ & 0,005 \\
\hline Manejo del ritmo inicial de parada & $3(20 \%)$ & $12(80 \%)$ & 0,025 \\
\hline Calidad de la RCP avanzada & $11(73 \%)$ & $13(87 \%)$ & NS \\
\hline Cuidados posparada & $5(33 \%)$ & $14(93 \%)$ & 0,0017 \\
\hline
\end{tabular}

a Prueba exacta de Fisher. NS: no significativo; RCP: reanimación cardiopulmonar.

te la primera $(p=0,0905)$ ni la segunda evaluación $(p=0,8804)$. Ningún sujeto fue excluido del estudio. El orden de participación de los sujetos de cada grupo durante las evaluaciones no demostró significación estadística al emplear la prueba de comparaciones múltiples de Tukey.

Se encontró que en las intervenciones y el abordaje de la RCP básica, así como en la calidad de la 
Figura. Mejoría en el logro de la competencia de reanimación cardiopulmonar avanzada.

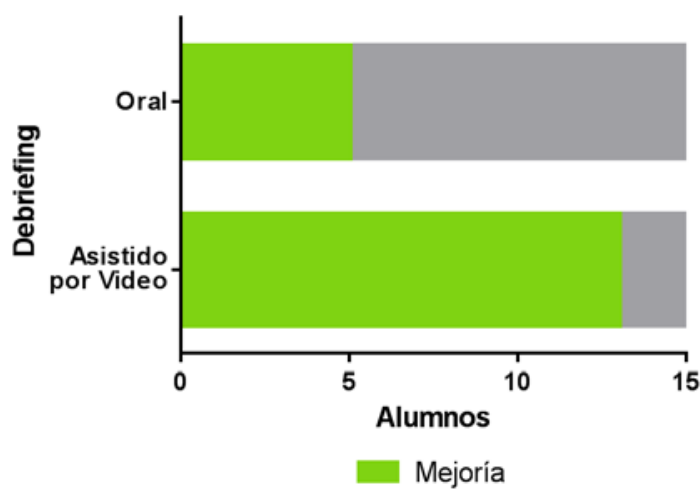

RCP en el grupo experimental, hubo una mayor proporción de alumnos que mejoraron tras tres prácticas que en el grupo control, pero no se encontró significación estadística en estos dos apartados. En evaluación del liderazgo, manejo del ritmo inicial de parada y manejo posparada se encontró una mejoría en el grupo experimental, con significación estadística (Tabla III). Los resultados de los ítems evaluados en las listas de comprobación de la competencia en RCP avanzada se presentan en las tablas IV y V.

Globalmente, al comparar desde la primera hasta la segunda evaluación en cuanto al logro de la competencia en RCP avanzada, se observó que mejoró significativamente en el grupo que recibió debriefing oral asistido por video, en comparación con el grupo que recibió únicamente debriefing oral (Figura).

\section{Discusión}

La eficacia de la adición del video a la técnica de enseñanza empleada en este estudio concuerda con lo comunicado por Birnbach et al, aunque difiere en que ellos encontraron una mejoría en el desarrollo de habilidades técnicas al observarse en video sin emplear debriefing, mientras que en este estudio, al emplear debriefing, la mejoría se observó en las habilidades no técnicas (liderazgo, manejo del ritmo inicial de parada y manejo posparada). Esto puede obedecer a la naturaleza del debriefing como técnica de enseñanza, que priorizó la reflexión so- bre lo no técnico (liderazgo, dominio del algoritmo, manejo del ritmo inicial de parada y cuidados posparada) $[13,14,20]$.

El impacto del debriefing asistido por video en la mejoría de habilidades específicas es congruente con lo comunicado por Grant et al, pero en su estudio no encontraron diferencias significativas en la mejoría global. Esto se atribuye al tiempo en el cual los estudiantes participaron en las sesiones de práctica (sólo cuatro horas), considerándolo escaso como para revelar diferencias entre ambos grupos, y a la diferencia de habilidades clínicas previas y experiencia que los estudiantes demostraron $[13,14,19]$.

En contraste, Savoldelli et al no encontraron diferencias significativas entre el empleo de debriefing oral y asistido por video. Esto podría relacionarse con el impacto del video en el contenido de la retroalimentación de los instructores, la sobrecarga de información adjudicable a la adición del video a la retroalimentación, la naturaleza distractora del video en los estudiantes -ocasionando que éstos prestaran menos atención a los comentarios constructivos y críticas del instructor- $y$, finalmente, un menor tiempo de instrucción y retroalimentación en el grupo que recibió retroalimentación con video $[13,14,18]$.

La mejoría observada en este estudio en el grupo experimental puede explicarse por las cualidades de la técnica de retroalimentación integral (debriefing) asistida por video empleada, en la que se procuró centrar la atención de los estudiantes en los comentarios y críticas en torno a momentos específicos a retroalimentar, evitando así proyecciones duraderas y distractoras $[13,18]$.

Otro factor relacionado puede ser el control sobre el número de escenarios, los tipos de escenarios y casos, y las condiciones académicas o trayectoria escolar de los estudiantes, que fueron uniformes. Además, todos los estudiantes se sometieron a una sesión para familiarizarse con las cualidades del simulador, los ritmos electrocardiográficos en el monitor y la dinámica de los escenarios de RCP (briefing) $[13,18,19,21,22]$.

Por otra parte, Coolen et al encontraron que la técnica de debriefing que seguía a la simulación asistida por video en tiempo real ofrece ventajas en el dominio de habilidades en estudiantes de pregrado. Esto es consistente con los resultados obtenidos en este estudio. La mejoría observada se debe a que esta estrategia incorpora elementos clave del aprendizaje del adulto: compromiso con el estudiante, vinculación con aprendizaje previo y oportunidad para la reflexión y retroalimentación. Además, la reproducción del video durante la retroalimenta- 
Tabla IV. Resultados de la evaluación del grupo que recibió debriefing asistido por video.

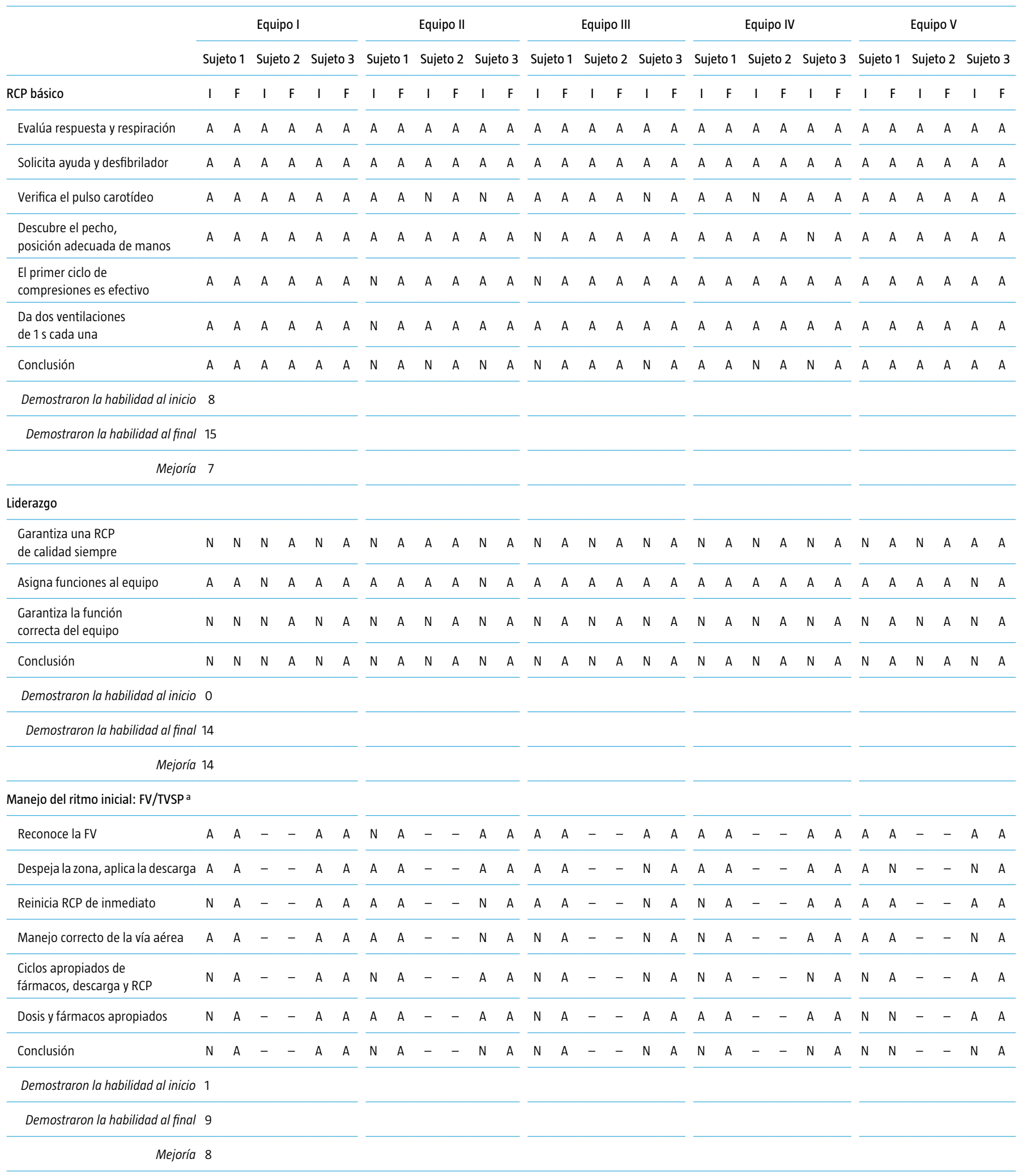


Tabla IV. Resultados de la evaluación del grupo que recibió debriefing asistido por video (cont.).

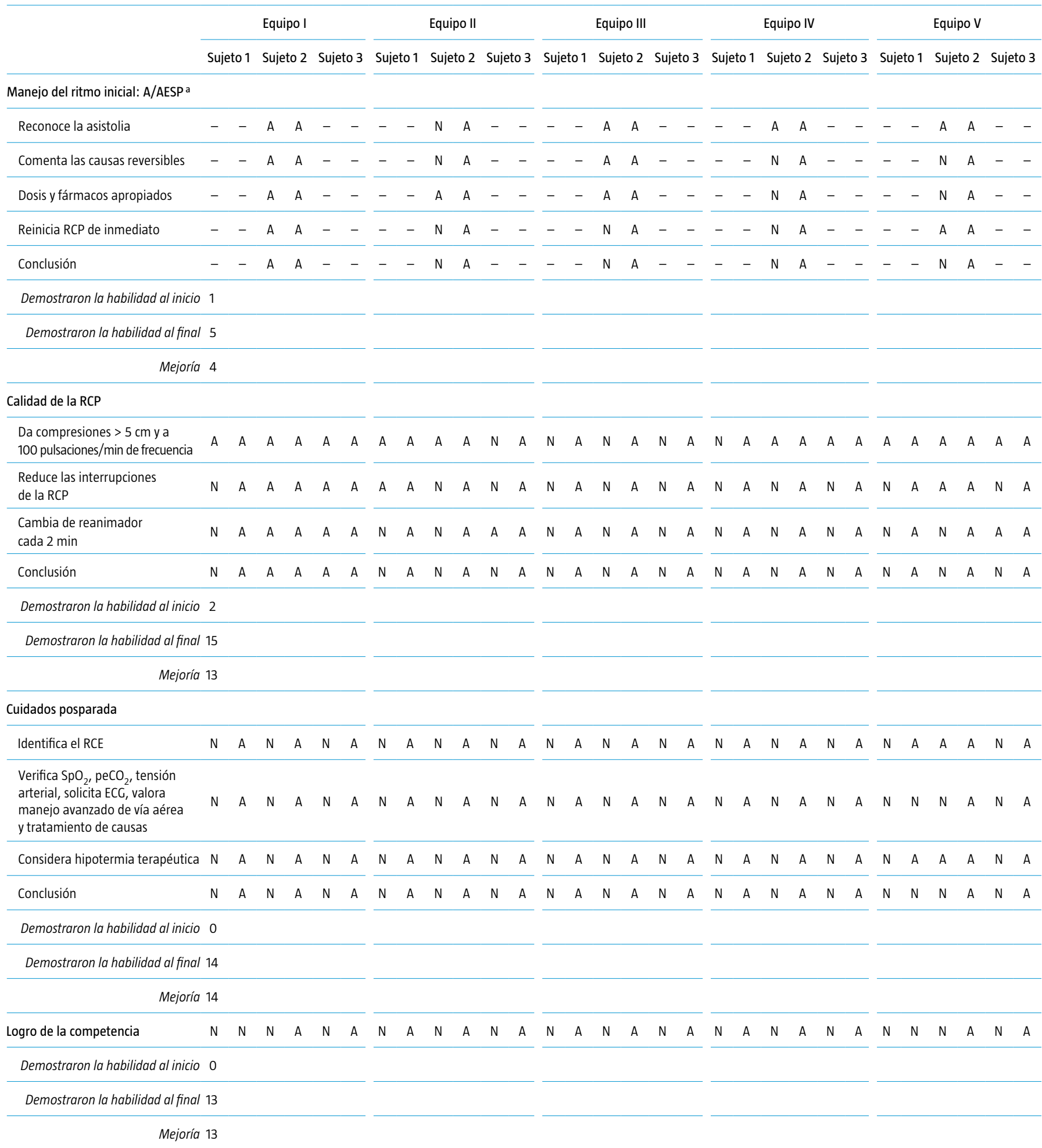

a Las puntuaciones de los apartados 'manejo de FV/TVSP' y 'manejo de A/AESP' constituyen uno solo: 'manejo del ritmo inicial de parada'. I: evaluación inicial; A: demuestra la habilidad; F: evaluación final; N: no demuestra la habilidad. 
Tabla V. Resultados de la evaluación del grupo que recibió debriefing oral.

\begin{tabular}{|c|c|c|c|c|c|c|c|c|c|c|c|c|c|c|c|c|c|c|c|c|c|c|c|c|c|c|c|c|c|c|}
\hline RCP básico & \multicolumn{6}{|c|}{ Equipo VI } & \multicolumn{6}{|c|}{ Equipo VII } & \multicolumn{6}{|c|}{ Equipo VII } & \multicolumn{6}{|c|}{ Equipo IX } & \multicolumn{6}{|c|}{ Equipo $X$} \\
\hline Evalúa respuesta y respiración & A & A & N & A & $A$ & A & $\mathrm{N}$ & A & A & $A$ & A & A & A & A & A & A & A & $A$ & A & A & A & A & A & A & A & $A$ & A & A & $\mathrm{N}$ & A \\
\hline Solicita ayuda y desfibrilador & A & A & A & A & $A$ & A & A & A & A & $A$ & A & A & A & A & A & A & A & A & A & $A$ & A & A & N & A & A & A & A & A & A & A \\
\hline $\begin{array}{l}\text { Descubre el pecho, } \\
\text { posición adecuada de manos }\end{array}$ & A & $A$ & $\mathrm{~N}$ & A & A & A & A & A & A & $A$ & $A$ & A & A & A & A & $A$ & A & A & A & $A$ & A & A & A & A & A & A & A & $\mathrm{N}$ & $\mathrm{N}$ & A \\
\hline El primer ciclo de compresiones es efectivo & $\mathrm{N}$ & A & $A$ & A & A & A & $\mathrm{N}$ & A & A & $A$ & A & A & A & A & A & A & A & A & $\mathrm{N}$ & $A$ & A & A & A & A & $A$ & A & A & A & A & A \\
\hline Da dos ventilaciones de $1 \mathrm{~s}$ cada una & A & A & $\mathrm{N}$ & A & A & $A$ & $A$ & A & A & $A$ & A & A & A & A & A & A & A & A & A & $A$ & A & A & A & A & $A$ & A & $A$ & A & $A$ & $A$ \\
\hline
\end{tabular}

Demostraron la habilidad al inicio 9

Demostraron la habilidad al final 12

Mejoría 3

Liderazgo

\begin{tabular}{|c|c|c|c|c|c|c|c|c|c|c|c|c|c|c|c|c|c|c|c|c|c|c|c|c|c|c|c|c|c|c|}
\hline Garantiza una RCP de calidad siempre & N & A & $\mathrm{N}$ & $A$ & $\mathrm{~N}$ & A & $\mathrm{N}$ & A & $\mathrm{N}$ & A & N & A & N & $\mathrm{N}$ & $\mathrm{N}$ & $\mathrm{N}$ & $A$ & $\mathrm{~N}$ & $\mathrm{~N}$ & $\mathrm{~N}$ & A & $\mathrm{N}$ & $\mathrm{N}$ & N & N & A & $\mathrm{N}$ & $\mathrm{N}$ & $\mathrm{N}$ & N \\
\hline Conclusión & $\mathrm{N}$ & $A$ & $\mathrm{~N}$ & $A$ & $\mathrm{~N}$ & A & $\mathrm{N}$ & $\mathrm{N}$ & $\mathrm{N}$ & A & N & A & $N$ & $\mathrm{~N}$ & $\mathrm{~N}$ & $\mathrm{~N}$ & A & $\mathrm{N}$ & $\mathbb{N}$ & $N$ & $\mathrm{~N}$ & $\mathbb{N}$ & $N$ & $\mathrm{~N}$ & $\mathrm{~N}$ & $\mathrm{~N}$ & $N$ & $N$ & $\mathrm{~N}$ & $\mathrm{~N}$ \\
\hline
\end{tabular}
Demostraron la habilidad al inicio 1 Demostraron la habilidad al final 5

Mejoría 4

Manejo del ritmo inicial: FV/TVSPa

\begin{tabular}{|c|c|c|c|c|c|c|c|c|c|c|c|c|c|c|c|c|c|c|c|c|c|c|c|c|c|c|c|c|c|c|}
\hline Reconoce la FV & $\mathrm{N}$ & $A$ & - & - & A & A & A & A & - & - & A & $A$ & A & $\mathrm{N}$ & - & - & $A$ & $A$ & $\mathrm{~N}$ & $\mathrm{~N}$ & - & - & A & A & A & A & - & - & $A$ & $\mathrm{~N}$ \\
\hline Despeja la zona, aplica la descarga & $A$ & $A$ & - & - & $\mathrm{N}$ & A & A & A & - & - & $A$ & $A$ & A & $\mathrm{N}$ & - & - & $A$ & $A$ & $\mathrm{~N}$ & $\mathrm{~N}$ & - & - & $\mathrm{N}$ & A & $\mathrm{N}$ & $\mathrm{N}$ & - & - & $\mathrm{N}$ & $\mathrm{N}$ \\
\hline Reinicia RCP de inmediato & $\mathrm{N}$ & $A$ & - & - & A & A & $A$ & $A$ & - & - & $A$ & $A$ & $A$ & $\mathrm{~N}$ & - & - & $A$ & $A$ & $\mathrm{~N}$ & $A$ & - & - & $A$ & A & $A$ & $A$ & - & - & $A$ & A \\
\hline Manejo correcto de la vía aérea & $\mathrm{N}$ & $A$ & - & - & A & A & $\mathrm{N}$ & A & - & - & $\mathrm{N}$ & $A$ & $A$ & $\mathrm{~N}$ & - & - & $\mathrm{N}$ & $A$ & $A$ & $\mathrm{~N}$ & - & - & $A$ & A & $\mathrm{N}$ & $\mathrm{N}$ & - & - & $A$ & $\mathrm{~N}$ \\
\hline $\begin{array}{l}\text { Ciclos apropiados de fármacos, } \\
\text { descarga y RCP }\end{array}$ & $\mathrm{N}$ & A & - & - & N & A & $\mathrm{N}$ & A & - & - & A & A & $A$ & $\mathrm{~N}$ & - & - & $A$ & $\mathrm{~N}$ & $\mathrm{~N}$ & A & - & - & $\mathrm{N}$ & A & A & $\mathrm{N}$ & - & - & $\mathrm{N}$ & A \\
\hline Dosis y fármacos apropiados & $\mathrm{N}$ & $A$ & - & - & A & A & $A$ & $A$ & - & - & $A$ & $A$ & A & $\mathrm{N}$ & - & - & $A$ & A & $A$ & $A$ & - & - & A & A & $A$ & $A$ & - & - & $A$ & A \\
\hline Conclusión & $\mathrm{N}$ & $A$ & - & - & $\mathrm{N}$ & A & $\mathrm{N}$ & $A$ & - & - & $\mathrm{N}$ & $A$ & $A$ & $\mathrm{~N}$ & - & - & $\mathrm{N}$ & $\mathrm{N}$ & $\mathrm{N}$ & $\mathrm{N}$ & - & - & $\mathrm{N}$ & A & $\mathrm{N}$ & $\mathrm{N}$ & - & - & $\mathrm{N}$ & $\mathrm{N}$ \\
\hline
\end{tabular}

Demostraron la habilidad al inicio 1

Demostraron la habilidad al final 5

Mejoría 4 
Tabla V. Resultados de la evaluación del grupo que recibió debriefing oral (cont.).

$\begin{array}{llll}\text { Equipo VI } & \text { Equipo VII } & \text { Equipo VII } & \text { Equipo IX }\end{array}$

Sujeto 1 Sujeto 2 Sujeto 3 Sujeto 1 Sujeto 2 Sujeto 3 Sujeto 1 Sujeto 2 Sujeto 3 Sujeto 1 Sujeto 2 Sujeto 3 Sujeto 1 Sujeto 2 Sujeto 3

Manejo del ritmo inicial: A/AESPa

\begin{tabular}{|c|c|c|c|c|c|c|c|c|c|c|c|c|c|c|c|c|c|c|c|c|c|c|c|c|c|c|c|c|c|c|}
\hline Reconoce la asistolia & - & - & $A$ & A & - & - & - & - & $A$ & $A$ & - & - & - & - & $A$ & $A$ & - & - & - & - & $A$ & A & - & - & - & - & $A$ & $A$ & - & - \\
\hline Comenta las causas reversibles & - & - & A & A & - & - & - & - & $\mathrm{N}$ & $A$ & - & - & - & - & $\mathrm{N}$ & $A$ & - & - & - & - & $A$ & A & - & - & - & - & $A$ & $A$ & - & - \\
\hline Dosis y fármacos apropiados & - & - & $A$ & A & - & - & - & - & $A$ & $A$ & - & - & - & - & $\mathrm{N}$ & $\mathrm{N}$ & - & - & - & - & $A$ & A & - & - & - & - & $A$ & $A$ & - & - \\
\hline Reinicia RCP de inmediato & - & - & $A$ & A & - & - & - & - & A & A & - & - & - & - & $A$ & $A$ & - & - & - & - & $A$ & $A$ & - & - & - & - & $A$ & $A$ & - & - \\
\hline Conclusión & - & - & A & A & - & - & - & - & $\mathrm{N}$ & $A$ & - & - & - & - & $\mathrm{N}$ & $\mathrm{N}$ & - & - & - & - & $A$ & A & - & - & - & - & $A$ & $A$ & - & - \\
\hline \multicolumn{31}{|c|}{ Demostraron la habilidad al inicio 3} \\
\hline \multicolumn{31}{|c|}{ Demostraron la habilidad al final 4} \\
\hline \multicolumn{31}{|c|}{ Mejoría 1} \\
\hline \multicolumn{31}{|l|}{ Calidad de la RCP } \\
\hline $\begin{array}{l}\text { Da compresiones }>5 \mathrm{~cm} \text { y a } \\
100 \text { pulsaciones/min de frecuencia }\end{array}$ & $\mathrm{N}$ & A & A & $A$ & A & $A$ & $\mathrm{~N}$ & $A$ & $\mathrm{~N}$ & A & A & $A$ & A & $A$ & A & A & A & A & A & $\mathrm{N}$ & $A$ & A & A & A & A & A & $A$ & A & $\mathrm{N}$ & A \\
\hline Reduce las interrupciones de la RCP & $\mathrm{N}$ & $A$ & $\mathrm{~N}$ & A & A & $A$ & A & $A$ & A & A & A & A & A & $A$ & A & A & A & A & $\mathrm{N}$ & $A$ & $A$ & A & $\mathrm{N}$ & A & A & A & A & A & A & A \\
\hline Cambia de reanimador cada 2 min & $\mathrm{N}$ & $A$ & $\mathrm{~N}$ & $A$ & A & $A$ & $\mathrm{~N}$ & $A$ & $\mathrm{~N}$ & A & A & A & $\mathrm{N}$ & $A$ & $\mathrm{~N}$ & A & $\mathrm{N}$ & $A$ & $\mathrm{~N}$ & $A$ & $\mathrm{~N}$ & A & $\mathrm{N}$ & A & A & $A$ & $\mathrm{~N}$ & A & $\mathrm{N}$ & A \\
\hline Conclusión & $\mathrm{N}$ & $A$ & $\mathrm{~N}$ & $A$ & $A$ & $A$ & $\mathrm{~N}$ & $A$ & $\mathrm{~N}$ & $A$ & $A$ & $A$ & $\mathrm{~N}$ & $A$ & $\mathrm{~N}$ & $A$ & $\mathrm{~N}$ & A & $\mathrm{N}$ & $\mathrm{N}$ & $\mathrm{N}$ & A & $\mathrm{N}$ & $A$ & $A$ & $A$ & $\mathrm{~N}$ & $A$ & $\mathrm{~N}$ & A \\
\hline
\end{tabular}

Demostraron la habilidad al inicio 3

Demostraron la habilidad al final 14

Mejoría 11

Cuidados posparada

\begin{tabular}{|c|c|c|c|c|c|c|c|c|c|c|c|c|c|c|c|c|c|c|c|c|c|c|c|c|c|c|c|c|c|c|}
\hline Identifica el RCE & $\mathrm{N}$ & $A$ & $\mathrm{~N}$ & $A$ & $\mathrm{~N}$ & $A$ & $\mathrm{~N}$ & $\mathrm{~N}$ & $\mathrm{~N}$ & $A$ & $A$ & A & $\mathrm{N}$ & $\mathrm{N}$ & $\mathrm{N}$ & $A$ & $\mathrm{~N}$ & $A$ & $\mathrm{~N}$ & $\mathrm{~N}$ & $\mathrm{~N}$ & $A$ & $\mathrm{~N}$ & $\mathrm{~N}$ & $A$ & A & A & $\mathrm{N}$ & $\mathrm{N}$ & $\mathrm{N}$ \\
\hline $\begin{array}{l}\text { Verifica } \mathrm{SpO}_{2}, \text { peCO}_{2} \text {, tensión arterial, } \\
\text { solicita } \mathrm{ECG} \text {, valora manejo avanzado } \\
\text { de vía aérea y tratamiento de causas }\end{array}$ & $\mathrm{N}$ & $A$ & $\mathrm{~N}$ & $A$ & $\mathrm{~N}$ & A & $\mathrm{N}$ & $\mathrm{N}$ & $\mathrm{N}$ & A & $\mathrm{N}$ & A & $\mathrm{N}$ & $\mathrm{N}$ & $\mathrm{N}$ & $\mathrm{N}$ & $\mathrm{N}$ & $\mathrm{N}$ & $\mathrm{N}$ & $\mathrm{N}$ & $\mathrm{N}$ & $\mathrm{N}$ & $\mathrm{N}$ & $\mathrm{N}$ & $\mathrm{N}$ & $\mathrm{N}$ & A & $\mathrm{N}$ & $\mathrm{N}$ & $\mathrm{N}$ \\
\hline Considera hipotermia terapéutica & $\mathrm{N}$ & $A$ & $\mathrm{~N}$ & $A$ & $\mathrm{~N}$ & A & $\mathrm{N}$ & $\mathrm{N}$ & $\mathrm{N}$ & A & $\mathrm{N}$ & A & $\mathrm{N}$ & $\mathrm{N}$ & $\mathrm{N}$ & $\mathrm{N}$ & $\mathrm{N}$ & $\mathrm{N}$ & $\mathrm{N}$ & $\mathrm{N}$ & $\mathrm{N}$ & $\mathrm{N}$ & $\mathrm{N}$ & $\mathrm{N}$ & $\mathrm{N}$ & $\mathrm{N}$ & $\mathrm{N}$ & $\mathrm{N}$ & $\mathrm{N}$ & $\mathrm{N}$ \\
\hline Conclusión & $\mathrm{N}$ & $A$ & $\mathrm{~N}$ & $A$ & $\mathrm{~N}$ & $A$ & $\mathrm{~N}$ & $\mathrm{~N}$ & $\mathrm{~N}$ & $A$ & $\mathrm{~N}$ & $A$ & $\mathrm{~N}$ & $\mathrm{~N}$ & $\mathrm{~N}$ & $\mathrm{~N}$ & $\mathrm{~N}$ & $\mathrm{~N}$ & $\mathrm{~N}$ & $\mathrm{~N}$ & $\mathrm{~N}$ & $\mathrm{~N}$ & $\mathrm{~N}$ & $\mathrm{~N}$ & $\mathrm{~N}$ & $\mathrm{~N}$ & $\mathrm{~N}$ & $\mathrm{~N}$ & $\mathrm{~N}$ & $\mathrm{~N}$ \\
\hline
\end{tabular}

Demostraron la habilidad al inicio 0

Demostraron la habilidad al final 5

Mejoría 5

Logro de la competencia

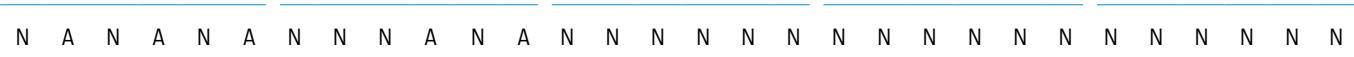

Demostraron la habilidad al inicio 0

Demostraron la habilidad al final 5

Mejoría 5

a Las puntuaciones de los apartados 'manejo de FV/TVSP' y 'manejo de A/AESP' constituyen uno solo: 'manejo del ritmo inicial de parada'. I: evaluación inicial; A: demuestra la habilidad; F: evaluación final; N: no demuestra la habilidad. 
ción podría ser útil para añadir perspectiva a la simulación al permitir a los alumnos ver cómo realmente participaron en vez de pensar o 'creer' cómo participaron $[13,15,21]$.

Debe destacarse que la mejoría con la adición de video a la retroalimentación se observó en los dos estudios que emplearon estudiantes de pregrado. Los resultados positivos encontrados en ambos estudios puede obedecer a que las condiciones académicas presentadas entre los sujetos resulten equiparables y sean dadas por el currículo académico $[13,15]$.

La falta de significación en los aspectos técnicos evaluados (intervenciones y evaluación de RCP básica y calidad de la RCP) entre el grupo experimental y el grupo control se relaciona con el nivel del dominio de estos aspectos en los estudiantes desde la primera sesión y la falta de un sistema de retroalimentación objetivo incluido en el simulador, por lo que no se pudo explorar a fondo la calidad de las compresiones durante las sesiones de retroalimentación integral oral y asistida por video, ni el énfasis de la técnica del debriefing en los aspectos no técnicos sobre los técnicos $[13,15,18]$.

Debe reconocerse que este estudio cuenta con ciertas limitaciones, como la selección no probabilística de la muestra, el tamaño pequeño de ésta y la diversidad encontrada en la técnica del debriefing, que varía de facilitador a facilitador, siendo más un arte que una ciencia. No existen suficientes estudios que comparen la ventaja de una técnica sobre otra y los resultados con los que se cuenta actualmente son contradictorios. Tampoco se dispone de ningún estudio que compare las diferencias de su uso en estudiantes de pregrado y posgrado. Estudios posteriores deben examinar estos aspectos en la simulación $[18,19,22]$.

Los resultados observados demuestran la ventaja de la técnica de la retroalimentación integral (debriefing) asistida por video en las habilidades no técnicas y en el dominio de la competencia de RCP avanzada en los estudiantes de pregrado de medicina.

\section{Bibliografía}

1. Galindo J, Visbal L. Simulación, herramienta para la educación médica. Salud Uninorte 2007; 23: 79-95.

2. Ponle G. An evaluation of David Kolb's theory of learning styles. Ilorin Journal of Education 2004; 23: 1-8.

3. Ten Eyck R, Tews M, Ballester J. Improved medical student satisfaction and test performance with a simulation-based emergency medicine curriculum: a randomized controlled trial. Ann Emerg Med 2009; 54: 684-91.
4. Miotto H, Ribeiro da Silva F, Valério C, Goulart E, Vieira M. Efecto en la resucitación cardiopulmonar utilizando entrenamiento teórico versus entrenamiento teórico-práctico. Arq Bras Cardiol 2010; 95: 328-31.

5. Rodgers DL, Securro S, Pauley RD. The effect of high-fidelity simulation on educational outcomes in an advanced cardiovascular life support course. Simul Healthc 2009; 4: 200-6.

6. Wayne DB, Butter J, Siddall VJ, Fudala MJ, Linquist LA, Feinglass J, et al. Simulation-based training of internal medicine residents in advanced cardiac life support protocols: a randomized trial. Teach Learn Med 2005; 17: 210-6.

7. Hoadley TA. Learning advanced cardiac life support: a comparison study of the effects of low- and high-fidelity simulation. Nurs Educ Perspect 2009; 30: 91-5.

8. Ward P, Johnson LA, Mulligan NW, Ward MC, Jones DL. Improving cardiopulmonary resuscitation skills retention: effect of two checklists designed to prompt correct performance. Resuscitation 1997; 34: 221-5.

9. Sutton RM, Donoghue A, Myklebust H, Srikantan S, Byrne A, Priest M, et al. The Voice Advisory Manikin (VAM): an innovative approach to pediatric lay provider basic life support skill education. Resuscitation 2007; 75: 161-8.

10. Kern KB, Stickney RE, Gallison L, Smith RE. Metronome improves compression and ventilation rates during CPR on a manikin in a randomized trial. Resuscitation 2010; 81: 206-10.

11. Bhanji F, Mancini M, Sinz E, Rodgers D, McNeil M, Hoadley T, et al. Part 16: education, implementation, and teams. 2010 American Heart Association Guidelines for Cardiopulmonary Resuscitation and Emergency Cardiovascular Care. Circulation 2010; 122: S920-33.

12. Palés J, Gomar C. El uso de las simulaciones en educación médica. Revista Electrónica de Teoría de la Educación. Educación y Cultura en la Sociedad de la Información 2010; 11: 147-69.

13. Fanning R, Gaba D. The role of debriefing in simulation-based learning. Simul Healthc 2007; 2: 115-25.

14. Cant R, Cooper S. The benefits of debriefing as formative feedback in nurse education. Aust J Adv Nurs 2011; 29: 37-47.

15. Raemer D, Anderson M, Cheng A, Fanning R, Nadkarni V, Savoldelli G. Research regarding debriefing as part of the learning process. Simul Healthc 2011; 6: S52-7.

16. Scherer LA, Chang MC, Meredith JW, Battistella FD. Videotape review leads to rapid and sustained learning. Am J Surg 2003; 185: 516-20.

17. Byrne AJ, Sellen AJ, Jones JG, Aitkenhead AR, Hussain S, Gilder F, et al. Effect of videotape feedback on anaesthetists performance while managing simulated anaesthetic crises: a multicentre study. Anaesthesia 2002; 57: 169-82.

18. Savoldelli GL, Naik VN, Park J, Joo HS, Chow R, Hamstra SJ. Value of debriefing during simulated crisis management: oral versus video-assisted oral feedback. Anesthesiology 2006; 105: 279-85.

19. Grant J, Moss J, Epps C, Watts P. Using video-facilitated feedback to improve student performance following highfidelity simulation. Clinical Simulation in Nursing 2010; 6: e177-84.

20. Birnbach D, Santos A, Bourlier R, Meadows W, Datta S, Stein D, et al. The effectiveness of video technology as an adjunct to teach and evaluate epidural anesthesia performance skills. Antesthesiology 2002; 96: 5-9.

21. Coolen E, Draaisma J, Hogeveen M, Antonius T, Lommen C, Loeffen J. Effectiveness of high fidelity video-assisted real-time simulation: a comparison of three training methods for acute pediatric emergencies. Int J Pediatr 2012; 2012: 709569.

22. Welke T, LeBlanc V, Savoldelli G, Joo H, Chandra D, Crabtree N, et al. Personalized oral debriefing versus standardized multimedia instruction after patient crisis simulation. Anesth Analg 2009; 109: 183-9, 